\title{
Effective Behavior of Nonlinear Microperiodic Composites with Imperfect Contact via the Asymptotic Homogenization Method
}

\author{
R. DÉCIO JÚNIOR ${ }^{1}$, L. D. PÉREZ-FERNÁNDEZ ${ }^{2}$ and J. BRAVO-CASTILLERO ${ }^{3}$
}

Received on February 15,2020 / Accepted on October 19, 2020

\begin{abstract}
The asymptotic homogenization method is applied here to one-dimensional boundary-value problems for nonlinear differential equations with rapidly oscillating piecewise-constant coefficients which model the behavior of nonlinear microperiodic composites, in order to assess the influence of interfacial imperfect contact on the effective behavior. In particular, a nonlinear power-law flux on the gradient of the unknown was considered. Several calculations were performed and are discussed at the end of this work, including a comparison of some results with variational bounds, which is also an important approach of this work.
\end{abstract}

Keywords: nonlinear composites, asymptotic homogenization method, imperfect contact.

\section{INTRODUCTION}

Composite materials can be described like heterogeneous materials formed by the union of two or more homogeneous ones, that will be their phases. These materials can be manufactured artificially or finded in nature (for example, wood and bone tissue). The capability to obtain better physical or chemical properties by the combination of different substances, made the composite materials one of the hotspot research topics in the modern technology [17].

In this work, will be considered periodic composites wich the phases distribution scale (microscopic scale) are simultaneously much larger than the atomic scale and much smaller than the macroscopic scale, characterizing a microperiodicity. Thus, the mathematical problems that modelling the physical behavior of these materials will present rapidly oscillating coefficients, wich

\footnotetext{
*Corresponding author: Roberto Décio Jr. - E-mail: roberto.decio.jr@gmail.com

${ }^{1}$ Programa de Pós Graduação em Modelagem Matemática, IFM, Universidade Federal de Pelotas, Campus Universitário, s/n, 96160-000, Capão do Leão, RS, Brasil. - E-mail: roberto.decio@ufpel.edu.br https://orcid.org/0000-0002-27667396

${ }^{2}$ Departamento de Matemática e Estatística, IFM, Universidade Federal de Pelotas, Campus Universitário, s/n, 96160000, Capão do Leão, RS, Brasil. - E-mail: leslie.fernandez@ufpel.edu.br https://orcid.org/0000-0002-4452-264X

${ }^{3}$ Departamento de Matemática y Mecánica, IIMAS, Universidad Nacional Autónoma de México, Delegación Álvaro Obregón, Apartado Postal 20-126, 01000 México City, México - E-mail: julian@mym.iimas.unam.mx https://orcid.org/0000-0002-7499-3821
} 
makes very difficult the application of standard numerical methods [13]. However, this characteristic satisfying the equivalent homogeneity hypothesis, However, this characteristic satisfying the Equivalent Homogeneity hypothesis, so the heterogeneous material can be considered physically equivalent to a homogeneous one, wich mathematical problems contain constants coefficients and its solution is satisfactorily close to the original problem's solution. The process to obtain this homogeneous material is called homogenization.

Among the mathematical homogenization methods is the asymptotic homogenization method (AHM) [2], wich consider a asymptotic expansion like an approximation of the solution from the problem of interest, in the form of a potential series of a small positive parameter that will be denoted by $\varepsilon$, which characterize the microscale. From applying this asymptotic expansion in the original problem, is obtained a recurrent sequence of problems for the coeficients of the $\varepsilon$ potences, from what one has locals problems, wich will give the terms of the asymptotic approach. The AHM have so many advantages, but the main is: the low computational need for the numerical methods to solve the problems involved in the recurrent sequence, and the good approximations of the exact solution of the original problem obtained. This application of the AHM has been very successfully, for example in [6]. Besides approximate the original solution, the AHM is applied to obtain the effective behavior of the heterogeneous material by the homogeneous equivalent one, that will be the application in focus on this paper.

In the field of mathematical models for physical phenomena, are finded linear constitutive laws, like the Hooke's law for the elasticity theory, the Fourie's law for the heat flow case, Nerst's law for the particle flux in gas, among other. However, there are so many physical phenomena of nonlinear nature, which ones can't be modeled by linear models, for example: plasticity, viscoelasticity, hyperelasticity, electrostriction, magnetostriction, thermoplasticity, etc [16]. Therefore, is very important the study of these models, expecting to obtain so prominent results how much the obtained in the linear case.

Lastly, in research about composites, another very important fact is the adhesion between its phases. Several works considered that the materials which constitute the composite had perfect binding, implying in the continuity of the solution of the problem that modeling a phenomenon in this materials [3]. But in the real situation the adhesion is not perfect, occurring mechanical imperfections, thermic isolations, chemical reactions or electric potential $[9,12]$. Because of this, is important consider imperfect contact conditions in the mathematical problems referent to this materials. In fact, it has been confirmed in the literature, where results showing the influence of the imperfect adhesion in composites $[1,9,10,15,18]$.

In that way, this paper shows how the AHM can evalueting the effective behavior of biphasics and microperiodc composites, applying the method in boundary values problems with the static heat diffusion equation, onedimensional, considering a relevant case of nonlineaity and comparing the situations of perfect and imperfect adhesions between its phases. Besides that, a brief approach of variational principles $[14,16]$ to obtain bounds to compare with the results obtained by AHM is given. This hybrid approach (AHM plus variational bounds, for imperfect contact composites) is rare to be founded in the current literature, similar results can be verified in [11]. 


\section{PROBLEM'S FORMULATION}

In practice, a ondedimensional composite can be considered like a bar. Without generality lost, will be considered a $\varepsilon$-periodic and biphasic bar, with unitary length, represented by $[0,1]$. The composite can be also represented by the finite union of its $N \in \mathbb{N}$ periodic cells, wich ones will be formed by the union of domains $\Omega_{1}$ and $\Omega_{2}$, wich represent the phases of the composite. Besides that, the boundaries $\partial \Omega_{1}$ and $\partial \Omega_{2}$ will be the interface points in the periodic cells, denoted by the $x_{j}$, and $\Gamma^{\varepsilon}=\left\{x_{j}\right\}_{j=1, \ldots, N}$ is the set of these interface points. More exactly,

$$
[0,1]=\bigcup_{i=1}^{N}\left(\Omega_{2 i-1} \cup \Omega_{2 i}\right) \cup\left(\partial \Omega_{2 i-1} \cup \partial \Omega_{2 i}\right) .
$$

The problem which modelling the diffusion phenomenon in this bar, which solution is $u^{\varepsilon}$, denoted by $P_{O}$, are formed as follows:

$$
\begin{array}{r}
\frac{d}{d x}\left[\sigma^{\varepsilon}\left(\frac{d u^{\varepsilon}}{d x}\right)\right]=f(x), x \in(0,1) / \Gamma^{\varepsilon} \\
\left.\llbracket \sigma^{\varepsilon}\left(\frac{d u^{\varepsilon}}{d x}\right)\right|_{x=x_{j}}=0 \\
\llbracket u^{\varepsilon}(x) \rrbracket_{x=x_{j}}=\left.\frac{\varepsilon}{\beta} \sigma_{1}^{\varepsilon}\left(\frac{d u^{\varepsilon}}{d x}\right)\right|_{x=x_{j}} \\
\left.u^{\varepsilon}\right|_{x=0}=g_{1},\left.u^{\varepsilon}\right|_{x=1}=g_{2},
\end{array}
$$

where the index $\varepsilon$ indicates the variable's dependence on the small parameter. More exactly, it indicates the dependence on the microscale, defined by the position variable $y=x / \varepsilon$, being $x$ the macroscale position variable (for example, $F^{\varepsilon} \equiv F(x / \varepsilon)$. Moreover, the flux $\sigma$ is expressed as:

$$
\sigma^{\varepsilon}\left(\frac{d u^{\varepsilon}}{d x}\right)=\left\{\begin{array}{c}
\sigma_{1}^{\varepsilon}\left(\frac{d u^{\varepsilon}}{d x}\right), x \in \Omega_{1} \\
\sigma_{2}^{\varepsilon}\left(\frac{d u^{\varepsilon}}{d x}\right), x \in \Omega_{2}
\end{array},\right.
$$

i.e., have one constitutive relation for each phase. The symbol $\llbracket \cdot \rrbracket_{x=x_{j}}$ indicates the continuity (or contact) condition in the interface points, defined as follows:

$$
\llbracket F^{\varepsilon}(x) \rrbracket=\lim _{x \rightarrow x_{j}^{+}} F^{\varepsilon}(x)-\lim _{x \rightarrow x_{j}^{-}} F^{\varepsilon}(x) \equiv F^{\varepsilon}\left(x_{j}^{+}\right)-F^{\varepsilon}\left(x_{j}^{-}\right) .
$$

The positive parameter $\beta$ is refered to imperfect contact condition, and can be interpreted as a condutance in the interface, in the order that $1 / \beta$ can be understood as a resistence. Notice that $\beta \rightarrow \infty$, the jump for $u^{\varepsilon}$ in (2.4) equation is null, that represent a perfect adhesion of the phases (perfect contact).

\section{THE AHM METHODOLOGY}

To beginning, are considered a two-scale asymptotic expansion as:

$$
u^{(2)}(x, y)=v_{0}(x)+\varepsilon u_{1}(x, y)+\varepsilon^{2} u_{2}(x, y),
$$


as an approach of the original solution $u^{\varepsilon}$ of the $P_{O}$ problem. Are considered that $v_{0}(x), u_{k}(x, y) \in$ $C^{2}([0,1])$ for $k=1,2,1-$ periodics in $y$ scale.

Replacing $u^{(2)}(x, y)$ in (2.2) equation, and considering the flux $\sigma$ linearized by a Taylor polynomial in the point $\zeta=d v_{0} / d x+\partial u_{1} / \partial y$, are obtained that:

$$
\begin{aligned}
\frac{d}{d x}\left[\sigma\left(\frac{x}{\varepsilon}, \frac{d u^{(2)}}{d x}\right)\right]-f(x) & =\varepsilon^{-1}\left[\frac{\partial \sigma}{\partial y}(y, \zeta)\right]+\varepsilon^{0}\left[\frac{\partial \sigma}{\partial x}(y, \zeta)+\right. \\
& \left.+\frac{\partial}{\partial y}\left[\left(\frac{\partial u_{1}}{\partial x}+\frac{\partial u_{2}}{\partial y}\right) \frac{\partial \sigma}{\partial \varepsilon}(y, \zeta)\right]-f(x)\right]+O(\varepsilon)
\end{aligned}
$$

Notice that from here, are considered the scales separation ( $x$ and $y$ ). Following, in order to satisfy:

$$
\left|\frac{d}{d x}\left[\sigma\left(\frac{x}{\varepsilon}, \frac{d u^{(2)}}{d x}\right)\right]-f(x)\right|=O(\varepsilon),
$$

has taking $u_{2} \equiv 0$, to obtain the follow equation for $\varepsilon^{-1}$ from 3.2:

$$
\frac{\partial \sigma}{\partial y}\left(y, \frac{d v_{0}}{d x}+\frac{\partial u_{1}}{\partial y}\right)=0 .
$$

Applying $u^{(2)}(x, y)$ also in the (2.3), (2.4) and (2.5), amongst (3.4), are obtained one-parametric family of problems, denoted by $P_{L}^{\frac{d v_{0}}{d x}}$, which has as solution $u_{1}(x, y)$, with the equations:

$$
\begin{array}{r}
\frac{\partial \sigma}{\partial y}\left(y, \frac{d v_{0}}{d x}+\frac{\partial u_{1}}{\partial y}\right)=0, y \in(0,1) /\left\{c_{1}\right\} \\
\llbracket \sigma\left(y, \frac{d v_{0}}{d x}+\frac{\partial u_{1}}{\partial y}\right) \rrbracket_{y=c_{1}}=0 \\
\llbracket u_{1} \rrbracket_{y=c_{1}}=\left.\frac{1}{\beta} \sigma_{1}\left(y, \frac{d v_{0}}{d x}+\frac{\partial u_{1}}{\partial y}\right)\right|_{y=c_{1}} \\
u_{1}(0,0)=u_{1}(l, 0)=0,
\end{array}
$$

where $c_{1}$ represents the interface point of the periodic cell on the microscale. The existence and unity of the 1-periodic solution of $P_{L}^{\frac{d v_{0}}{d x}}$, are guaranteed by the Lemma 3.1, that is a generalization for the imperfect contact condition, from an analogous one (see [2]). 
Lemma 3.1 (Lemma). Let $\bar{\varepsilon}$ a parameter and $\sigma(y, \varepsilon)$ a piecewise and continuously differentiable function in $[0,1]$. Then, for all $x$ fixed, there are $\mathscr{N}_{1}(y, \bar{\varepsilon}) 1$-periodic in y functions, which solve the one-parametric family of problems $P_{L}^{\bar{\varepsilon}}$ with the parameter $\bar{\varepsilon}$, defined as follows:

$$
\begin{array}{r}
\frac{\partial \sigma}{\partial y}\left(y, \bar{\varepsilon}+\frac{\partial \mathscr{N}_{1}}{\partial y}\right)=0, y \in(0,1) /\left\{c_{1}\right\} \\
\llbracket \sigma\left(y, \bar{\varepsilon}+\frac{\partial \mathscr{N}_{1}}{\partial y}\right) \rrbracket_{y=c_{1}}=0 \\
\llbracket \mathscr{N}_{1} \rrbracket_{y=c_{1}}=\left.\frac{1}{\beta} \sigma_{1}\left(y, \bar{\varepsilon}+\frac{\partial \mathscr{N}_{1}}{\partial y}\right)\right|_{y=c_{1}} \\
\mathscr{N}_{1}(0, \bar{\varepsilon})=0 .
\end{array}
$$

The condition $\mathscr{N}_{1}(0, \bar{\varepsilon})=0$ guarantees the solution's unity.

Since the Lemma 3.1 is a original generalization of this work, the main idea of its demonstration will be given.

Proof. From the integration of (3.9) equation and the condition in (3.10) give:

$$
\sigma\left(y, \bar{\varepsilon}+\frac{\partial \mathscr{N}_{1}}{\partial y}\right)=\bar{\sigma},
$$

where $\bar{\sigma}$ is constant in relation to $y$.

By defining an auxiliary function $T(y, \varepsilon)=\sigma(y, \varepsilon)-\bar{\sigma}$, it follows that

$$
\frac{\partial T}{\partial \varepsilon}=\frac{\partial \sigma}{\partial \varepsilon},
$$

and by assuming $\partial T / \partial \varepsilon>0$ (fact that agree with the most physical interpretations), from the implicit function theorem [8], is possible to assert the existence of an inverse function $\varepsilon(y, \bar{\sigma})=$ $\bar{\varepsilon}+\partial \mathscr{N}_{1} / \partial y$, continuous and differentiable in a neighbourhood of the point $(y, \bar{\sigma})$, which is 1 -periodic in relation to $y$.

Thus, the integration $\partial \mathscr{N}_{1} / \partial y$ from $\varepsilon(y, \bar{\sigma})$ gives:

$$
\begin{array}{r}
\mathscr{N}_{1}(y, \bar{\varepsilon})=\int_{0}^{y}(\varepsilon(s, \bar{\varepsilon})-\bar{\varepsilon}) d s, y \in\left[0, c_{1}\right) \\
\mathscr{N}_{1}(y, \bar{\varepsilon})-\llbracket \mathscr{N}_{1} \rrbracket_{y=c_{1}}=\int_{0}^{y}(\varepsilon(s, \bar{\sigma})-\bar{\varepsilon}) d s . y \in\left(c_{1}, 1\right],
\end{array}
$$

The term $\llbracket \mathscr{N}_{1} \rrbracket_{y=c_{1}}$ in (3.16) equation appears because the improper integration that is needed in the $[0,1]$ interval, after the point $y=c_{1}$. Lastly, the solution $\mathscr{N}_{1}$ can be defined as:

$$
\mathscr{N}_{1}(y, \bar{\varepsilon})=\left\{\begin{array}{l}
\int_{0}^{y}(\varepsilon(s, \bar{\sigma})-\bar{\varepsilon}) d s, y \in\left[0, c_{1}\right) \\
\mathscr{N}_{1}\left(c_{1}^{-}\right)+\bar{\sigma} \bar{\beta}, y=c_{1} \\
\int_{0}^{y}(\varepsilon(s, \bar{\sigma})-\bar{\varepsilon}) d s+\frac{\bar{\sigma}}{\beta}, y \in\left(c_{1}, 1\right) \\
0, y=1
\end{array} .\right.
$$


It is interesting explain that the choice for $\left.\mathscr{N}_{1}\right|_{y=c_{1}}$ have as inspiration the Dirichlet theorem for Fourier's series [7].

The demonstration is finished with the prove of the 1-periodicity of $\mathscr{N}_{1}$, which is made showing that $\mathscr{N}_{1}(y+1, \varepsilon)-\mathscr{N}_{1}(y, \varepsilon)=0$ for every $y \in[0,1] /\left\{c_{1}\right\}$.

The unicity and existence of the solution of the family of problems $P_{L}^{\frac{d v_{0}}{d x}}$ in (3.5)-(3.8) equations, made possible the obtaining of the effective law (which means the effect physical behavior) for the composite of interest. It is defined by the functional relation between the the flux average $(\bar{\sigma})$ and the gradient of $v_{0}(\bar{\varepsilon})$.

As in the demonstration of the Lemma 3.1, from the integration of (3.5) equation and the condition in (3.6), follows that

$$
\sigma\left(y, \frac{d v_{0}}{d x}+\frac{\partial u_{1}}{\partial y}\right)=\bar{\sigma}
$$

and the integration of $\partial u_{1} / \partial y$ from (3.18) equation, gives:

$$
\frac{\partial u_{1}}{d y}=\varphi(y, \bar{\sigma})-\frac{d v_{0}}{d x},
$$

where $\varphi(y, \bar{\sigma})$ is the inverse function of the flux $\sigma$ in (3.18), which is guaranteed by the implicit function theorem.

Thus, applying the average operator $\langle$.$\rangle to (3.19), which is defined by:$

$$
\langle.\rangle=\int_{0}^{1}(.) d y
$$

yields:

$$
\left.u_{1}\right|_{y=1}-\llbracket u_{1} \rrbracket_{y=c_{1}}-\left.u_{1}\right|_{y=0}=\langle\varphi(y, \bar{\sigma})\rangle-\frac{d v_{0}}{d x} .
$$

From the contact condition in (3.7) and the 1-periodicity in relation to $y$ of $u_{1}$ (that is guaranteed by the Lemma 3.1), (3.21) equation turns to:

$$
\overline{\bar{\sigma}}=\langle\varphi\rangle(\bar{\sigma})-\frac{d v_{0}}{d x} .
$$

The effective law, which will denoted by $\hat{\sigma}\left(\frac{d v_{0}}{d x}\right)$, is obtained by the relation between $\bar{\sigma}$ and $d v_{0} / d x$, which is implicit in (3.22). In practice, is necessary to solve de equation like (3.22) for $\bar{\sigma}$, which resolution methods will depend of the form of the constitutive relations in each phase of composite.

\section{RESULTS OF AN APPLICATION}

\subsection{The composite considered}

Will be considered a biphasic composite, $\varepsilon$-periodic: one linear phase (phase 1) and another nonlinear (phase 2). The nonlinearity is of the potential type, with exponent $n>0$, which mod- 
elling several physical phenomena, like plastic deformation and thermoplasticity, generally with $n<1$ ( [16], [5]):

$$
\sigma^{\varepsilon}\left(\frac{d u^{\varepsilon}}{d x}\right)=\left\{\begin{array}{l}
K_{1} \frac{d u^{\varepsilon}}{d x}, x \in \Omega_{1} \\
K_{2}\left(\frac{d u^{\varepsilon}}{d x}\right)^{n}, x \in \Omega_{2}
\end{array}\right.
$$

where $K_{1}$ and $K_{2}$ are the coefficients, that in the physical meaning, are the individuals properties of each phase, like the elasticity modulus, electric and thermal conductivity, etc.

The application of the the AHM as is showing in the section 3.22 to the problem like $P_{O}$ formulated in the section 2, with the constitutive relation in (4.1), gives the equation:

$$
\frac{c_{1}}{K_{1}} \bar{\sigma}+\frac{c_{2}}{K_{2}^{1 / n}} \bar{\sigma}^{1 / n}+\frac{\bar{\sigma}}{\beta}-\bar{\varepsilon}=0,
$$

where $c_{1}$ can be interpreted also like the phase 1 concentration and e $c_{2}=1-c_{1}$, the phase 2 concentration. The terms of (4.2) equation, will be dimensionless by $K_{1}$, then the equation (4.2) becomes:

$$
\lambda^{1 / n} c_{2}\left(\bar{\sigma}_{0}\right)^{1 / n}+\left(c_{1}+\frac{1}{\beta_{0}}\right)\left(\bar{\sigma}_{0}\right)-\bar{\varepsilon}=0,
$$

where $\lambda=K_{1} / K_{2},\left(\bar{\sigma}_{0}\right)=\bar{\sigma} / K_{1}$ and $\beta_{0}=\beta / K_{1}$. The effective law for this composite will be determined by solving the equation (4.3) for $\bar{\sigma}_{0}$. For example, if $n=1 / 2$, (4.3) is a quadratic equation, and if $n=1 / 3,(4.3)$ is a cubic equation of the form $m^{3}+p m+q=0$ which can be solved by the Cardano-Tartáglia formula. For another values of $n$, is recommend numerical methods, as the classical Newton's iteration, which is used in this work.

\subsection{Some numerical simulations}

To obtain the effective law from (4.3), the Newton's iteration [4] was applied for different values for $n, c_{1}, \lambda$ and $\beta_{0}$. The implementation of the method was based on a tolerance of $10^{-5}$, and a initial point:

$$
\sigma_{*}=\frac{\bar{\varepsilon}}{2} \max \left\{\frac{1}{c_{1}}, \frac{1}{\lambda^{1 / n} c_{2}}\right\}
$$

because is possible to see that will exist a solution in the interval $\left[0, \sigma_{*}\right]$, for (4.3), by the bisection method logic.

In Figure 1, the perfect contact is considered, and the effective law is determined for $c_{1}=0.1$ and some values for $n(n \leq 1)$ and $\lambda$. The Figure 1 shows a pure linear behavior for $n=1$, which confirms that the effective behavior of a composite with two linear phases will be also linear (that can be proved analytically). Besides that, as $n$ is growing, the effective behavior lost its linear aspect, in both values of $\lambda$ considered. Notice that the case with $\lambda=2$ represents a family of composites which linear property twice mayor than the nonlinear one (that shows the linear phase like a a stronger phase), and the opposite for $\lambda=1 / 2$. 

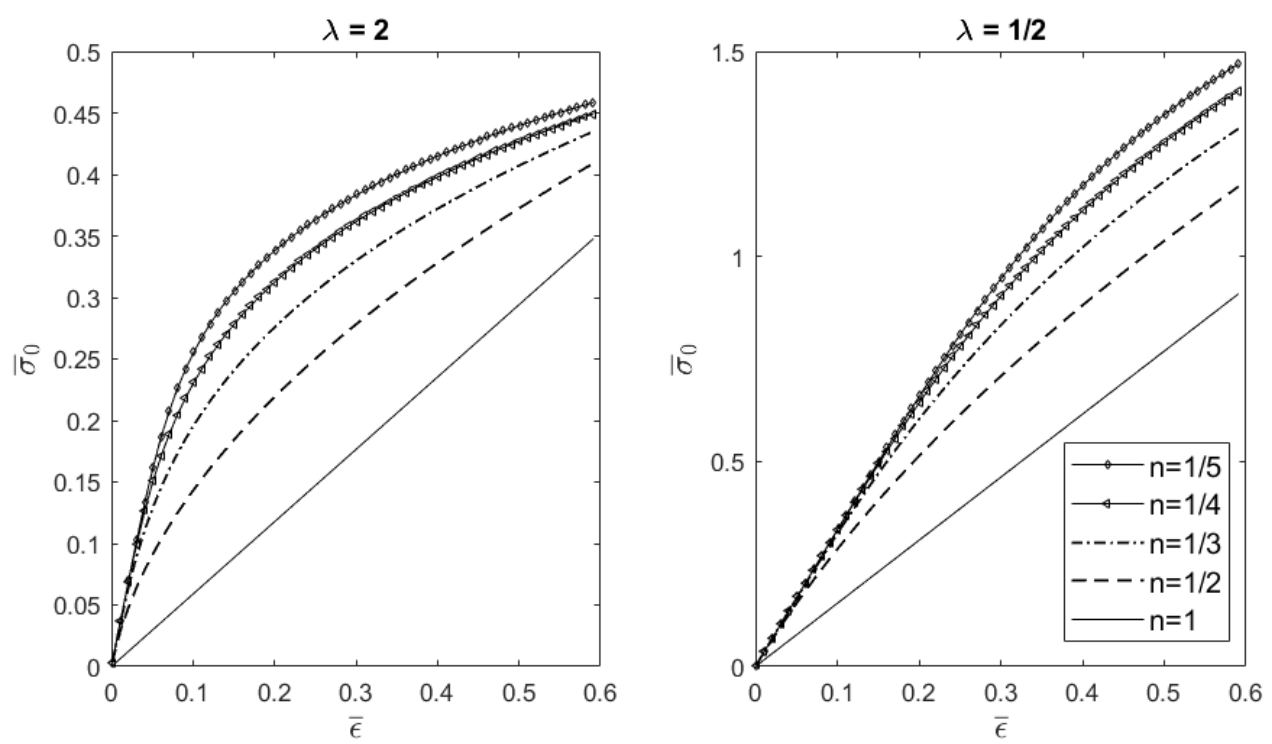

Figure 1: Effective law for the perfect contact condition, for some values of $n, c_{1}=0.1$ and fixed $\lambda$.

The effective behaviour obtained by de AHM for the perfect contact condition was compared to a upper bound (UB) wich is result of a combination of variational principals (see $[11,14,16]$ ). The result in Figure 2 is showing in a logaritmic scale for better visualization.

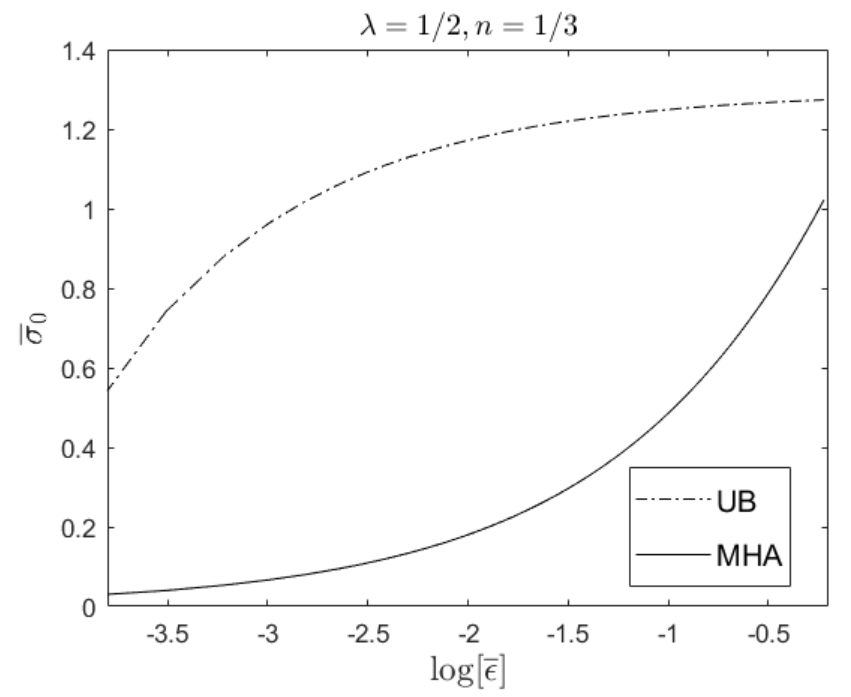

Figure 2: Comparison of the effective law for the perfect contact condition obtained by AHM and a upper bound (UB) following $[11,14,16])$. 
The Figure 2 shows what can be a shy validation of the results wich are obtained by AHM for the evaluated case. A better analisys and numerical implementation are necessary for a effective validation, including the imperfect contact case.
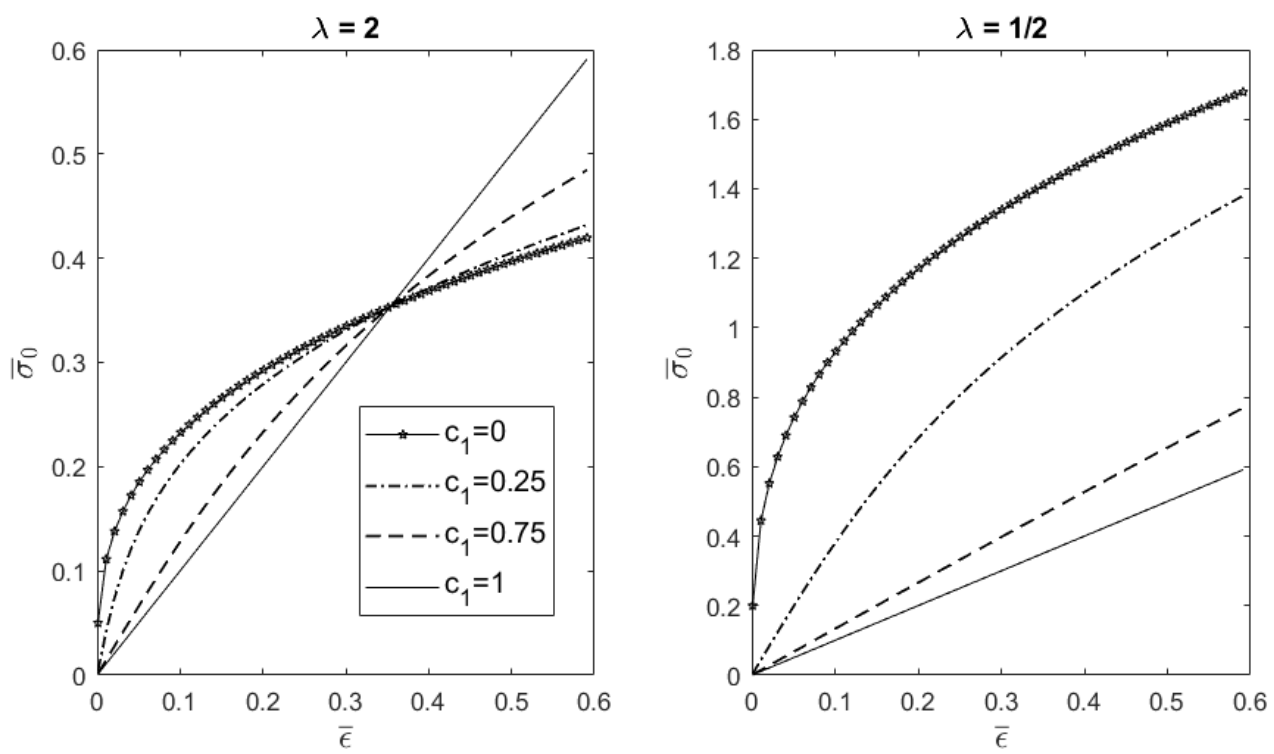

Figure 3: Effective law for the perfect contact condition, for $n=1 / 3$, fixed values of $\lambda$, and for different values of $c_{1}$.

The Figure 3 shows the effect behavior for the perfect contact again, with $n=1 / 3$, for some values of $c_{1}$ (which means that the composition of composite is varying). Notice that for both cases of $\lambda$, as the concentration $c_{1}$ increases, the effective behavior near of the linear behavior, and the opposite occurs as $c_{1}$ decreases. How the cases where $c_{1}=1$ e $c_{1}=0$ represent a pure material composed by linear and nonlinear material, respectively, this results show de influence of each material constituent on the effective behavior of the composite.

In the Figure 4 the effective behavior is evaluated for different cases of imperfect contact, with $c_{1}=0.3$ and $n=1 / 3$, varying the parameter $\beta_{0}$. It's possible to see the asymptotic behavior of the effective laws as $\beta_{0} \rightarrow \infty$, when the effective behavior for imperfect contact case nears the perfect contact one. In fact, the difference between the curves is imperceptible for $\beta_{0}>50$. Moreover, the effective laws seems to be approaching to a null behavior $(\hat{\sigma}(\bar{\varepsilon}) \equiv 0)$ as $\beta_{0} \rightarrow 0^{+}$. This last behaviour can be verified in a better way in the Figure 5 .

These results show the coherence of the effective behavior in the imperfect contact case. Thinking about physical meaning, like the conductivity phenomena for example, as smaller are the $\beta_{0}$ value, bigger is the resistance to the heat (or electric energy) in the interface of the composite, i.e., smaller the effective flux on the material. Besides that, as mayor is the $\beta_{0}$ value, the effective flux can't be greater than the flux in the contact perfect flux. 

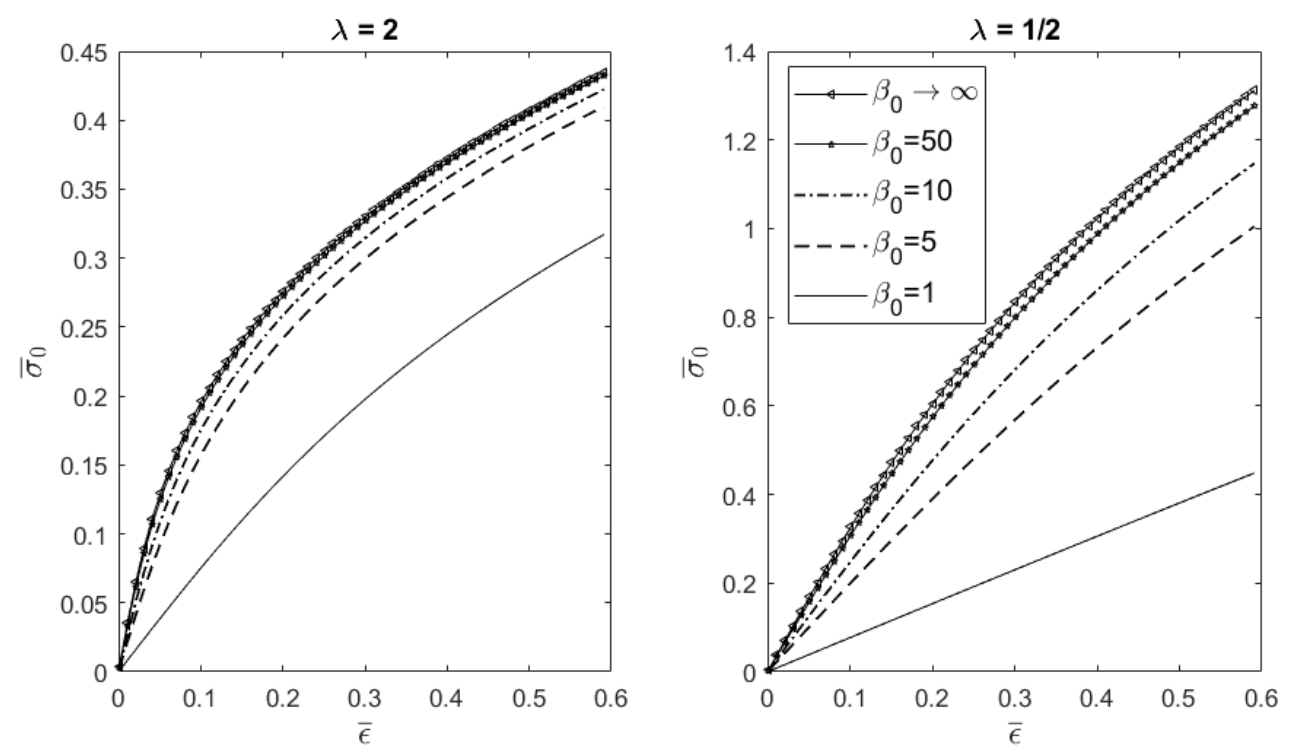

Figure 4: Effective law for the imperfect contact condition, for fixed values of $\lambda, n=1 / 3, c_{1}=$ 0.3 , and for different values of the parameter $\beta_{0}\left(\beta_{0} \rightarrow \infty\right)$.
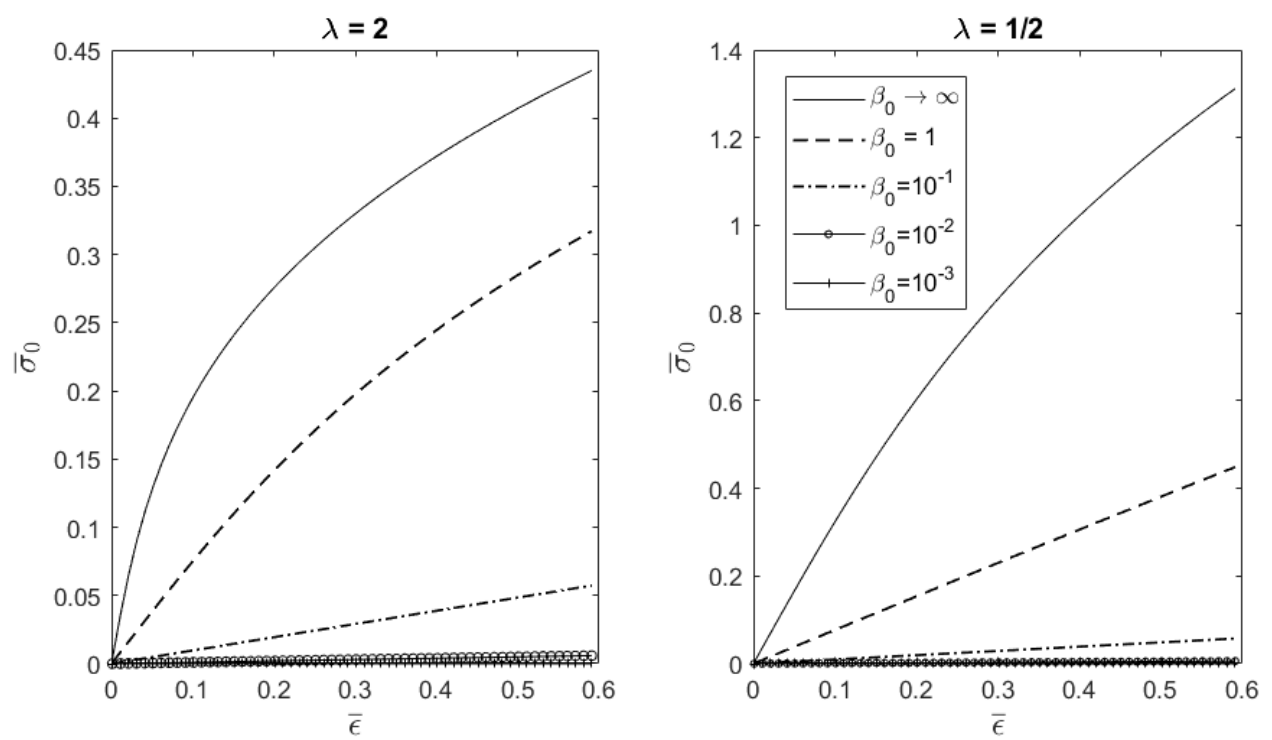

Figure 5: Effective law for the imperfect contact condition, for fixed values of $\lambda, n=1 / 3, c_{1}=$ 0.3 , and for different values of the parameter $\beta_{0}\left(\beta_{0} \rightarrow 0^{+}\right)$. 
In the elasticity context, the increase of the interface's resistance can be interpreted like the decrease of the rigidity on the interface. So, as smaller are the $\beta$, smaller are this rigidity, which results in the smaller effective rigidity of the composite. That is, the material will be more deformable, when it is submitted to the same tensions forces. It results in tension-deformation curves with smaller inclination, which is possible also to see in the Figure 4.

\section{CONCLUSIONS}

The AHM was successful in providing the effective law of nonlinear microperiodic composites with interfacial imperfect contact. Its application showed that variations of phase concentration, nonlinearity index and interfacial imperfection parameter affect importantly the effective behavior of the composite.

In this work was also possible to compare the result by AHM with other method wich is based in variational principles. But, better formulation and implementation of this other method are necessary to present effective validation of these results. Furthermore, this methodology has to be applied to the imperfect contact case too.

This and other types of nonlinearities, as well as other homogenization approaches, will be considered in future works in order to validate and extend the present one.

RESUMO. O método de homogenização assintótica é aplicado a problemas de valor de contorno unidimensionais para equações diferenciais não lineares com coeficientes constantes por partrs e rapidamente oscilantes que modelam o comportamento de compósitors microperiódicos não lineares, com o objetivo de avaliar a influência do contato imperfeito entre as fases no comportamento efetivo. Em particular, considerou-se um fluxo não linear de lei de potência no gradiente da incógnita. Vários resultados computacionais são apresentados e discutidos no final deste trabalho, incluindo uma comparação de alguns resultados com cotas variacionais, uma abordagem importante deste trabalho.

Palavras-chave: compósitos não lineares, método de homogeneização assintótica, contato imperfeito.

\section{REFERENCES}

[1] F.E. Álvarez-Borges, J. Bravo-Castillero, M.E. Cruz, R. Guinovart-Díaz, L.D. Pérez-Fernández, R. Rodríguez-Ramos \& F.J. Sabina. Reiterated homogenization of a laminate with imperfect contact: gain-enhancement of effective properties. Applied Mathematics and Mechanics, 39 (2018), 1119-1146.

[2] N.S. Bakhvalov \& G.P. Panasenko. "Homogenisation: Averaging Processes in Periodic Media". Kluwer Academic Publishers, Dordrecht (1989).

[3] Y. Benveniste. The effective mechanical behaviour of composite materials with imperfect contact between the constituents. Mechanics of Materials, 4 (1985), 197-208. 
[4] R. Burden, J.D. Faires \& A. Burden. "Análise Numérica”. CENGAGE Learning, São Paulo, SP/Brasil (2015).

[5] G. Dai, F. Jarrar, F. Ozturk \& J. JSheik-Ahmad. On the Effect of the Complexity of the Constitutive Model in Simulating Superplastic Forming. Defect and Diffusion Forum, 385 (2018), 379-384.

[6] R.M. Décio Júnior, L.D. Pérez-Fernández \& J. Bravo-Castillero. Exactness of formal asymptotic solutions of a Dirichlet problem modeling the steady state of functionally-graded microperiodic nonlinear rods. Journal of Applied Mathematics and Computational Mechanics, 18(3) (2019), 45-56.

[7] B. Demidovitch, G. Baranenkov, V. Efimenko, S. Frolov, S. Kogan, G. Luntz, E. Porshneva, R. Shostak, E. Sitcheva \& A. Yanpolski. "Problemas e Exercícios de An'alise Matem'atica". Mir, Moscou (1987).

[8] V.A. Ilyin \& E.G. Poznyak. "Fundamentals of Mathematical Analysis, Part 1". MIR, Moscou (1982).

[9] R. Lipton \& B. Vernescu. Composites with imperfect interface. Proceedings of the Royal Society, 452 (1996), 329-358.

[10] J.C. López-Realpozo, R. Rodríguez-Ramos, R. Guinovart-Díaz, J. Bravo-Castillero \& F.J. Sabina. Compuestos elásticos no lineales con condiciones de contacto imperfectas. Revista Electrónica Nova Scientia, 1(1) (2008), 53-64.

[11] J. López-Ruiz, J. Bravo-Castillero, R. Brenner, M. Cruz, R. Guinovart-Díaz, L.D. Pérez-Fernández \& R. Rodríguez-Ramon. Variational bounds in composites with nonuniform interfacial thermal resistance. Applied Mathematical Modelling, 39(23,24) (2015), 7266-7276.

[12] F.L. Neto. "Compósitos Estruturais”. Blucher, São Paulo (2012).

[13] G.P. Panasenko. Homogenization for Periodic Media: from Microscale to Macroscale. Physics of Atomic Nuclei, 71(4) (2008), 681-694.

[14] M. Peigney. A pattern-based method for bounding the effective response of a nonlinear composite. Journal of the Mechanics and Physics of solids, 53 (2005), 923-948.

[15] R. Penta \& A. Gerisch. The asymptotic homogenization elasticity tensor properties for composites with material discontinuities. Continuum Mechanics and Thermodynamics, 29 (2017), 187-206.

[16] P. Ponte-Castañeda \& P. Suquet. Nonlinear Composites. Advances in Applied Mechanics, 34 (1998), 171-303.

[17] D.K. Rajak, D.D. Pagar, R. Kumar \& C.I. Pruncu. Recent progress of reinforcement materials: a comprehensive overview of composite materials. Journal of Materials Research and Technology, 8(6) (2019), 6354-6374.

[18] S. Torquato \& M.D. Rintoul. Effect of the Interface on the Properties of Composite Media. Physical Review Letters, 75(22) (1995), 4067-4070. 